\title{
Study on Dynamic Strain Regularity and Influencing Factors of Shallow Buried Metal Pipe under Collapse Impact Load
}

\author{
Min Wang $\mathbb{D}^{1},{ }^{1}$ Mingshou Zhong $\left(\mathbb{D},{ }^{1}\right.$ Yuan Long $\mathbb{D}^{\mathbb{D}},{ }^{1}$ Kai Ding, ${ }^{2}$ Xingbo Xie, ${ }^{1}$ and Liu Ying ${ }^{1}$ \\ ${ }^{1}$ Academy of Field Engineering, Army Engineering University of PLA, Nanjing, Jiangsu 210007, China \\ ${ }^{2}$ Science and Technology on Near-Surface Detection Laboratory, Wuxi, Jiangsu 214035, China \\ Correspondence should be addressed to Mingshou Zhong; zhongms7@126.com
}

Received 17 April 2018; Revised 12 July 2018; Accepted 22 July 2018; Published 2 December 2018

Academic Editor: Nicola Nisticò

Copyright (c) 2018 Min Wang et al. This is an open access article distributed under the Creative Commons Attribution License, which permits unrestricted use, distribution, and reproduction in any medium, provided the original work is properly cited.

With the combination of model experiment and numerical simulation, we explore the effect of collapse height, weight, and pipesoil stiffness ratio on dynamic strain of shallow buried metal pipe under the collapse impact load. By analyzing the strain at different measuring points of the buried pipeline, the strain law of the buried pipeline under the collapse impact load is obtained. Based on the range analysis and variance significance analysis, it was found that the pipe-soil stiffness ratio has a more significant impact on the dynamic strain of the buried pipeline under impact compared to the collapse height and the weight. Then, the numerical simulation method was used to further analyze the effect of pipe-soil stiffness ratio on the dynamic response of buried pipelines; the following conclusions are drawn: As the stiffness ratio of pipe-soil increases, the plastic stress and strain of the buried pipeline will decrease, and influence of the pipeline by the collapse impact is slighter.

\section{Introduction}

With the construction of "China West to East Gas Transmission" and the continued advancement of urbanization, the coverage of oil and gas pipelines and other types of buried pipelines is rapidly expanding. Buried pipelines have the advantages of safety and high efficiency, saving urban space, and reducing ground accidents [1]. However, under the influence of various external forces, there are also many failures on buried pipelines such as pipeline rupture and breakage, leading to major safety accidents such as oil and gas leaks, fires, and explosions [2, 3]. Collapse impact load is one of the reasons that can easily cause pipeline damage $[4,5]$. With the acceleration of urban construction, a large number of urban buildings (structures) are facing demolition. And blasting method is one of the commonly used methods. Because the collapse vibration energy during the blasting demolition process is larger and the vibration frequency is closer to the natural vibration frequency of the building, the pipeline is more likely to be damaged under the collapse impact load. Therefore, it is of great social and economic significance to study the mechanical properties and structural stability of buried pipelines under collapse impact loads.

Many scholars have made a comprehensive study on the deflection and dynamic response of buried pipelines under external forces. Wang [6] studied the interaction between pipe and soil in buried pipelines and the influence of traffic loads on the static and dynamic response of buried pipelines. Yang [7] applied the Boussinesq method and ABAQUS software to analyze the dynamic response of buried pipelines under impact loads and to solve the internal forces and deformations of the pipelines. Sun [8] established the mechanical model of buried pipelines under the action of ground overload, analyzed the influence of pipeline depth, pipe stiffness, and other factors on the dynamic response parameter distribution of buried pipelines, and applied ABAQUS software to numerical simulation study. Liu and Yang [9] conducted a finite element analysis of the dynamic response of buried pipelines under impact loads and discussed the effects of various factors, such as pipe-soil stiffness ratio and pipeline depth, on the dynamic response of pipelines. Gresnigt and Karamanos [10] proposed an analytical model based on the shell theory to determine 
the elastoplastic deformation of buried pipelines under impact loads. At the same time, the finite element method was used for additional verification studies. Mosadegh and Nikraz [11] took the response of the buried pipeline under traffic load as background and carried out experiments and numerical simulations. The relationship between the strain stress of the pipeline and the soil displacement was obtained by numerical fitting. However, most of the previous studies used theoretical and numerical simulation methods, lacking test results to verify; besides, research on the impact of collapse impact loads on buried pipelines is not enough. In this paper, the influence of the collapse height, the mass of weight, and the pipe-soil stiffness ratio on the dynamic strain of the buried pipeline under the impact load of collapse is deeply studied using test methods combined with numerical simulation.

\section{Experimental Study}

2.1. Test Equipment and Design. The experiment was designed by reduction and scaling according to a large preexperiment of viaduct blasting demolition project which Pro.Long have participated. The preexperimental layout is shown in Figure 1. In the preexperiment, the inner diameter of the steel pipe is $70 \mathrm{~cm}$, the outer diameter is $72 \mathrm{~cm}$, the buried depth is $1 \mathrm{~m}$, the top layer of the pipeline is $40 \mathrm{~cm}$ backfilling fine sand and $60 \mathrm{~cm}$ road layer structure made of C15 concrete. The test sample in this paper was thin-walled cylindrical pipes which were made of Q235 steel with wall thickness of $3 \mathrm{~mm}$, outer diameter of $117 \mathrm{~mm}$, and length of $1.0 \mathrm{~m}$. The dropping hammer is also made of Q235 steel, and the mass of the three types of dropping hammers is $8 \mathrm{~kg}$, $16 \mathrm{~kg}$, and $32 \mathrm{~kg}$, respectively (Table 1 for specific size). The device layout of impact test on the buried pipeline is shown in Figure 2. Firstly, three deep pits with a length of $0.6 \mathrm{~m}$, a width of $1 \mathrm{~m}$, and a depth of $0.5 \mathrm{~m}$ were excavated on flattened land with an interval of $0.4 \mathrm{~m}$ between each pit. In order to explore the influence of different soil qualities on buried pipelines under the collapse impact, the pits are filled with fine sand, soil, and coarse sand, respectively. The buried depth of the metal pipe is $12 \mathrm{~cm}$. Two scaffolds with a height of $10 \mathrm{~m}$ are erected on both sides of the pits. Then a flat plate is set between them, and a pulley is suspended at the center of the flat plate. The falling hammer is lifted to a specified height to simulate the collapse impact. We can simulate a collapse impact by lifting the drop hammer to a specified height and then free fall with these devices.

In order to explore the stress-strain response of buried pipelines under the action of collapse impact loads, the BE120-4BA type universal strain flower produced by AVIC Electric Test Company was placed on the test steel pipe. The technical parameters are shown in Table 2. Four pieces of strained flowers were placed on each steel pipe in the test, and each strained flower contained two mutually perpendicular sensitive grids. Therefore, two vertical strains could be measured. Each strained flower was laid along the axial and circumferential directions of the pipe. The eight strain tests were numbered. The specific layout and numbering methods are shown in Figure 3.

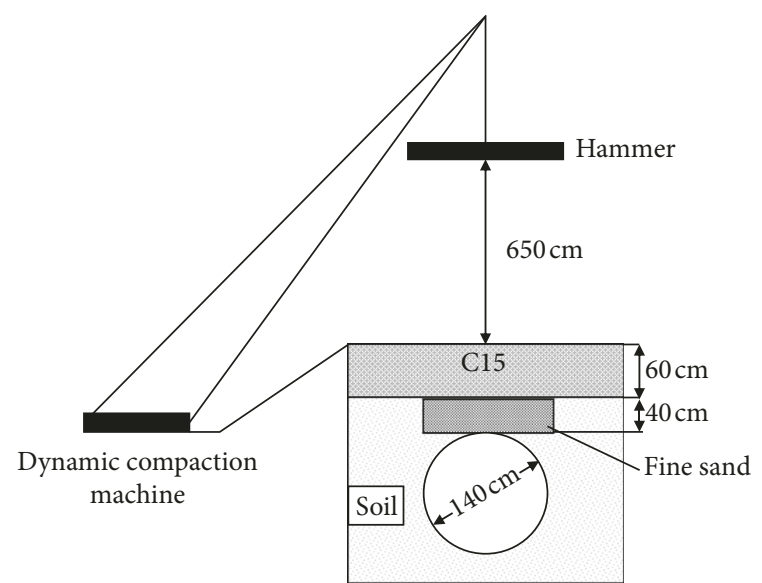

Figure 1: Preexperiment layout.

TABle 1: Mass and size of drop hammer.

\begin{tabular}{lccc}
\hline Mass $(\mathrm{kg})$ & Size $(\mathrm{cm})$ & \\
\hline 8 & Length & Width & Height \\
16 & 20 & 11.3 & 4.5 \\
32 & 30 & 15.1 & 4.5 \\
\hline
\end{tabular}

The purpose of this experiment was to investigate the influence of collapse height $M$, weight $H$, and soil conditions $R$ on the buried pipe under the collapse impact load. The experiment was designed as method of orthogonal test. There are three levels for each influencing factor. The mass of drop hammer has three levels $8 \mathrm{~kg}, 16 \mathrm{~kg}$, and $32 \mathrm{~kg}$. The height of fall is $4 \mathrm{~m}, 6 \mathrm{~m}$, and $8 \mathrm{~m}$. The pipe-soil stiffness ratio is calculated as follows:

$$
\alpha_{\mathrm{s}}=\frac{E_{\mathrm{p}}}{E_{\mathrm{d}}}\left(\frac{t}{r_{0}}\right)^{3},
$$

where $E_{\mathrm{p}}$ is the elastic modulus of the pipe, $\mathrm{MPa}$; $t$ is the wall thickness of the pipe, $\mathrm{mm} ; r_{0}$ is the calculated radius of the pipe, that is, the distance from the center of the pipe to the center of the pipe wall, $\mathrm{mm}$; and $E_{\mathrm{d}}$ is the resilience modulus of the backfill, MPa. The measurement of soil stiffness is aided by the PFWD (portable falling weight deflectometer). According to the following equation, the resilience modulus is determined:

$$
E_{\mathrm{p}}=\frac{2 \pi p a\left(1-\mu^{2}\right)}{4 l},
$$

where $p$ is the measured pressure of the load-bearing plate $(\mathrm{kPa}) ; a$ is the radius of the load-bearing plate, the loadbearing plate with a radius of $15 \mathrm{~cm}$ is used in the test; $\mu$ is the Poisson coefficient, take 0.35 ; and $l$ is the measured center plate deflection $(\mu \mathrm{m})$.

By calculation, the pipe-soil stiffness ratios for the three types of soil (compact soil, loose sand, and loose sand) in this experiment were $0.843,1.215$, and 1.602 , respectively. Then, nine kinds of test conditions were designed based on the orthogonal table of 3 factors and 3 levels. The specific test scheme is shown in Table 3. 


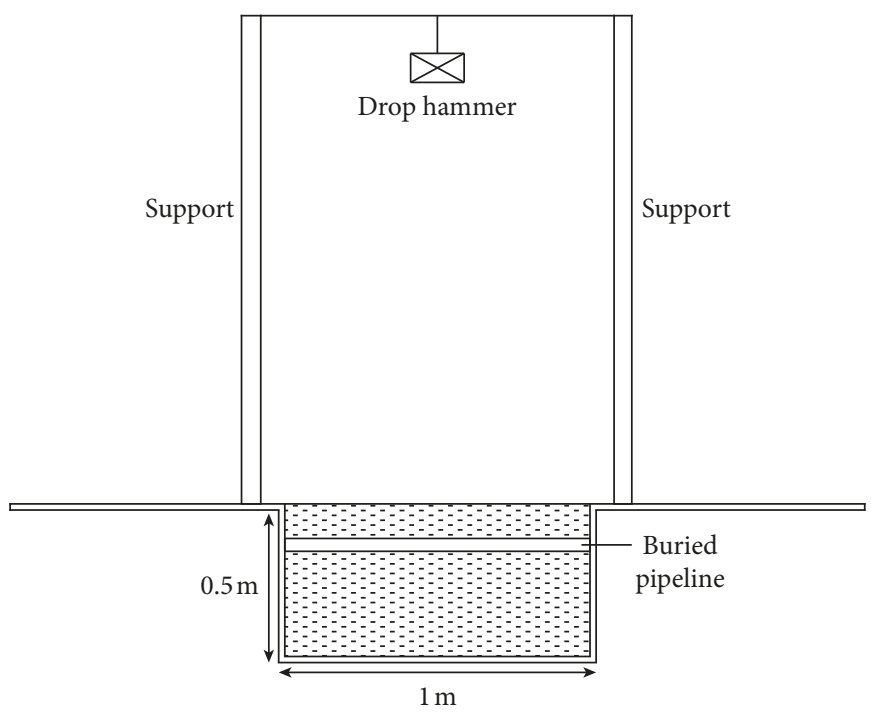

(a)

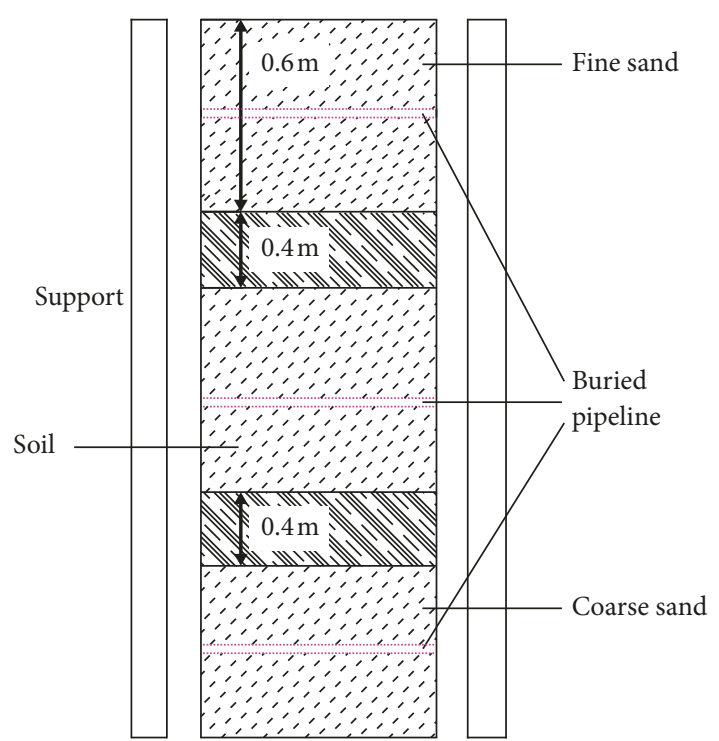

(b)

FIgURE 2: Schematic diagram of impact experiment of the buried pipeline. (a) Front view. (b) Top view.

TABLE 2: Strain gauge technical parameters.

\begin{tabular}{lcccc}
\hline Type & Sensitivity coefficient & Strain limit & Size of sensitive grids $(\mathrm{mm})$ & Size of footing $(\mathrm{mm})$ \\
\hline BE-120-4BA & 2.1 & $2 \%$ & $3.8 \times 1.7$ & $11.7 \times 11.7$ \\
\hline
\end{tabular}

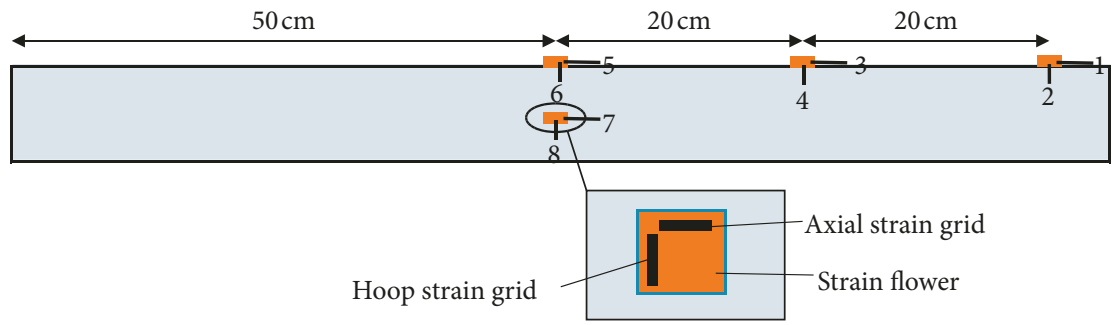

(a)

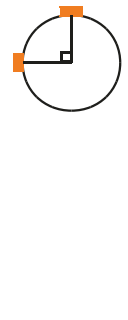

(b)

FIgURE 3: Schematic diagram of strain gauge layout. (a) Front view. (b) Side view.

TABLE 3: Impact experiment scheme of the buried pipeline.

\begin{tabular}{lccc}
\hline Condition & $\begin{array}{c}\text { Mass of } \\
\text { hammer }(M / \mathrm{kg})\end{array}$ & $\begin{array}{c}\text { Height of } \\
\text { collapse }(H / \mathrm{m})\end{array}$ & $\begin{array}{c}\text { Pipe-soil } \\
\text { stiffness ratio }(R)\end{array}$ \\
\hline 1 & 8 & 8 & 1.60 \\
$\mathbf{2}$ & $\mathbf{1 6}$ & $\mathbf{6}$ & $\mathbf{1 . 6 0 2}$ \\
3 & 32 & 4 & 1.602 \\
4 & 8 & 4 & 1.215 \\
$\mathbf{5}$ & $\mathbf{1 6}$ & $\mathbf{8}$ & $\mathbf{1 . 2 1 5}$ \\
6 & 32 & 6 & 1.215 \\
7 & 8 & 6 & 0.843 \\
$\mathbf{8}$ & $\mathbf{1 6}$ & $\mathbf{4}$ & $\mathbf{0 . 8 4 3}$ \\
9 & 32 & 8 & 0.843 \\
\hline
\end{tabular}

\subsection{Test Results and Analysis}

2.2.1. Study on Strain Law of Pipeline. According to the four strain flowers laid on the pipeline, the axial and circumferential strains of the corresponding four points on the pipeline were measured. According to the strain test results, the stress and strain of the buried pipeline under the collapse impact load can be analyzed. As shown in Figure 4, the strain data measured under the nine conditions of the experimental design are shown. The strain curve number in each figure corresponds to the number in Figure 3. Through the analysis of the strain curve of the pipeline, the strain situation on the pipeline under each operating condition is the same, that is, the closer to the center of the pipeline, the greater the pipeline strain. The strain on the measuring point (1-6 curves) on the strike face is negative and the maximum strain exceeds $-1000 \mu \varepsilon$, indicating that the point on the pipe is compressed and plastic strain occurs. The strains on the lateral points ( 7 and 8 curves) are basically positive, indicating that the pipe is stretched at this point. Through further observation, it can be found that the axial strain at each measuring point is less than the circumferential strain under 9 working conditions, indicating that the buried pipe 

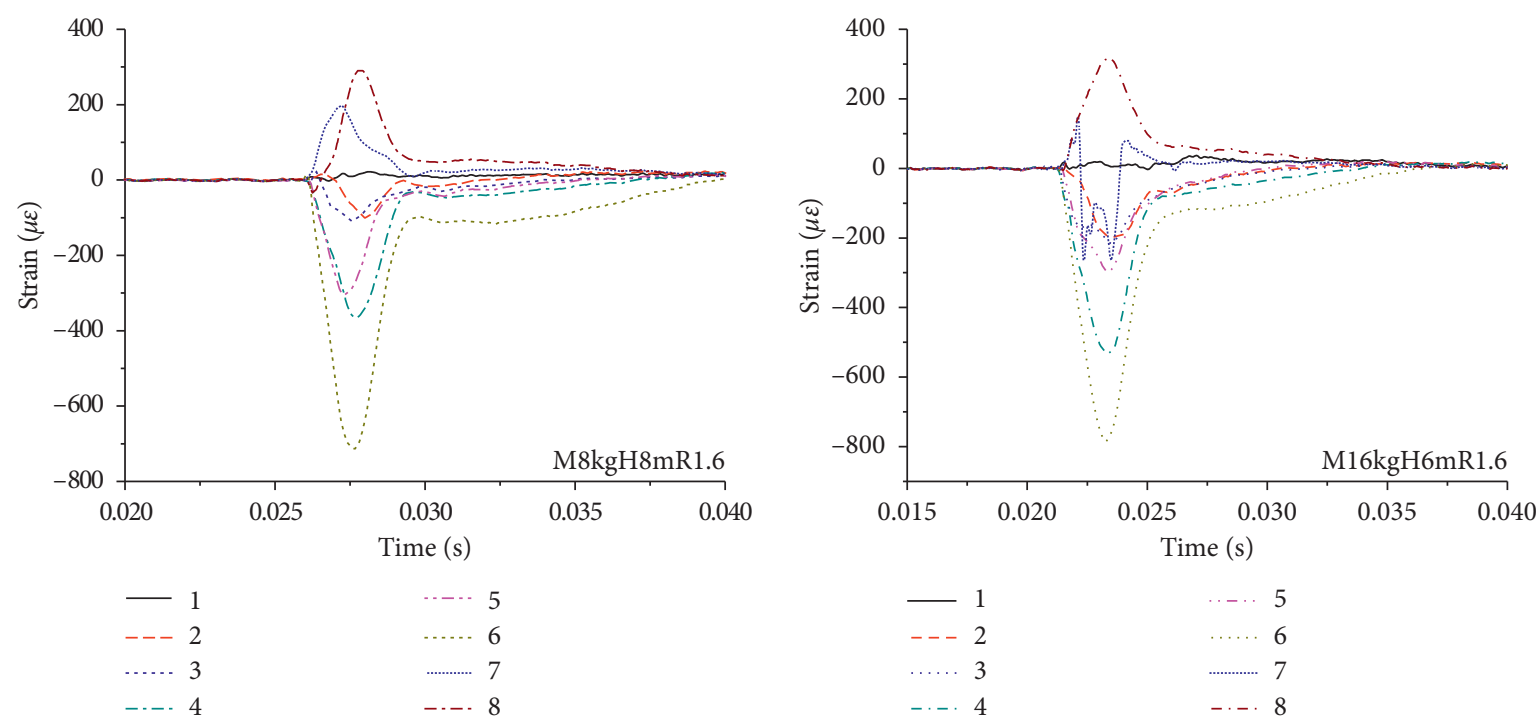

(a)
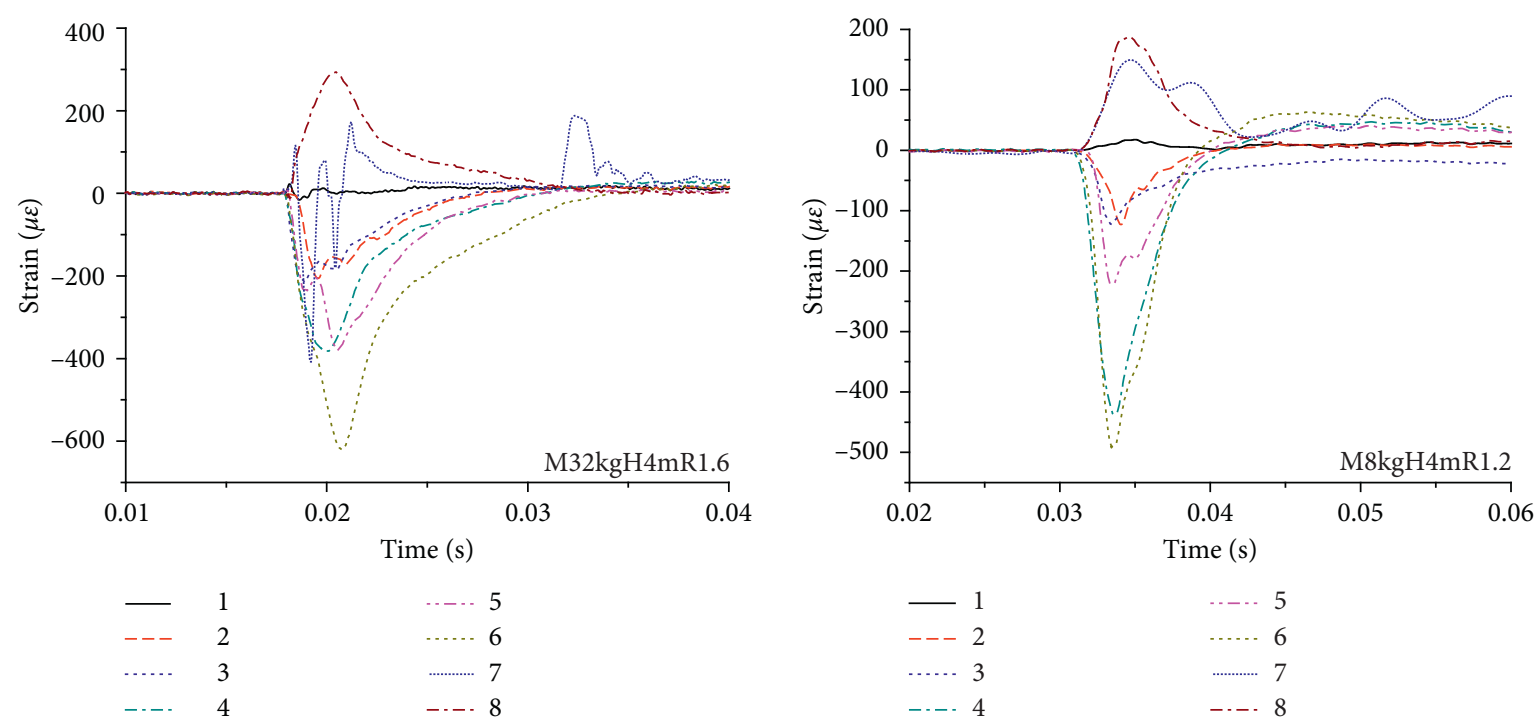

(c)
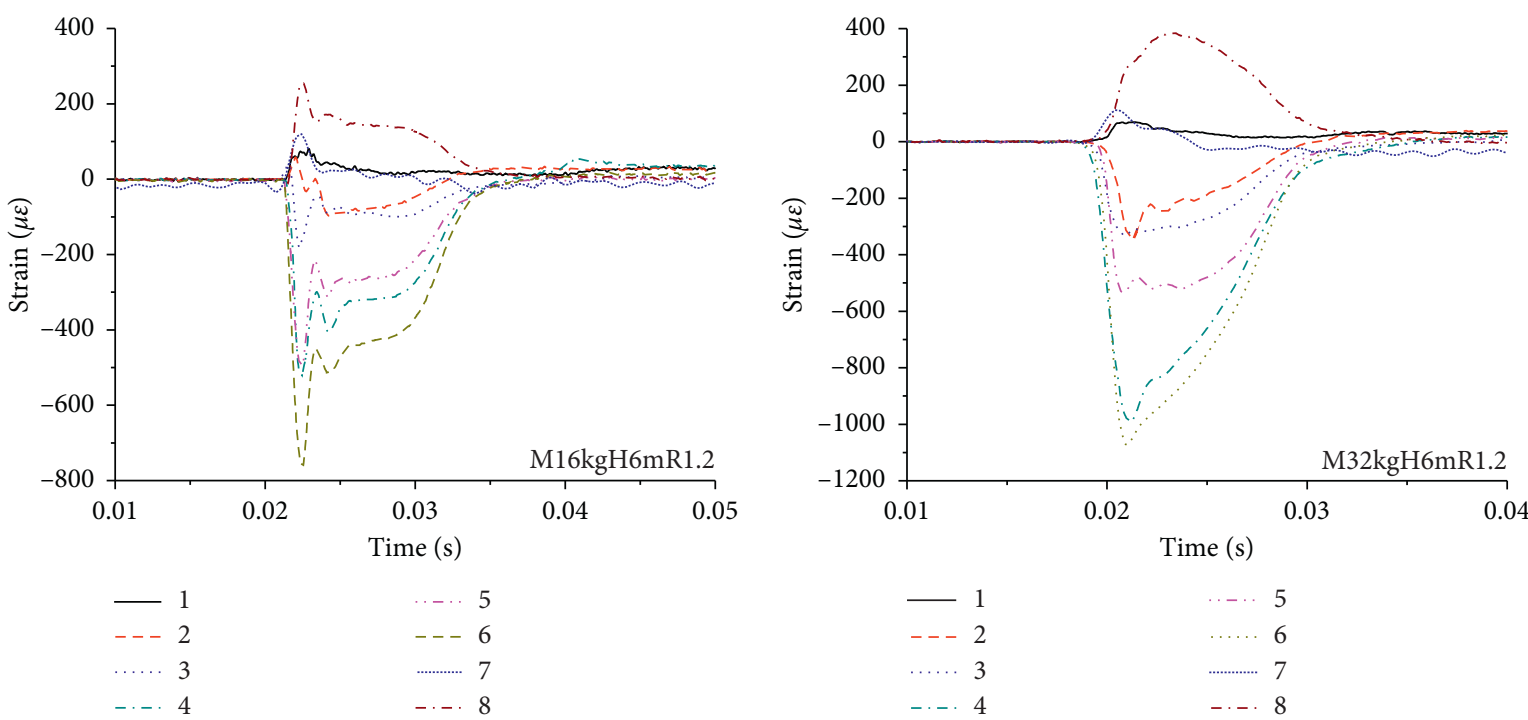

(e)

(f)

Figure 4: Continued. 


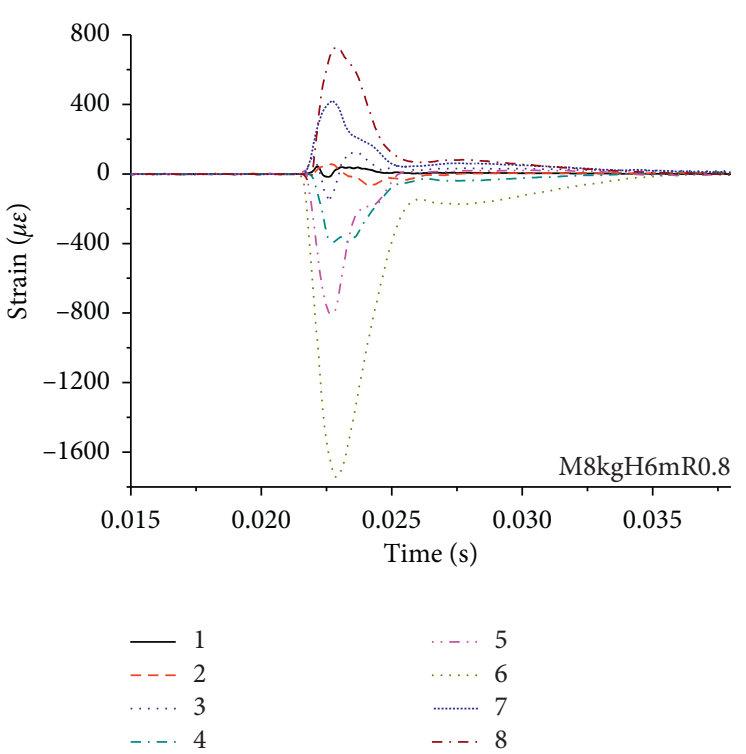

(g)

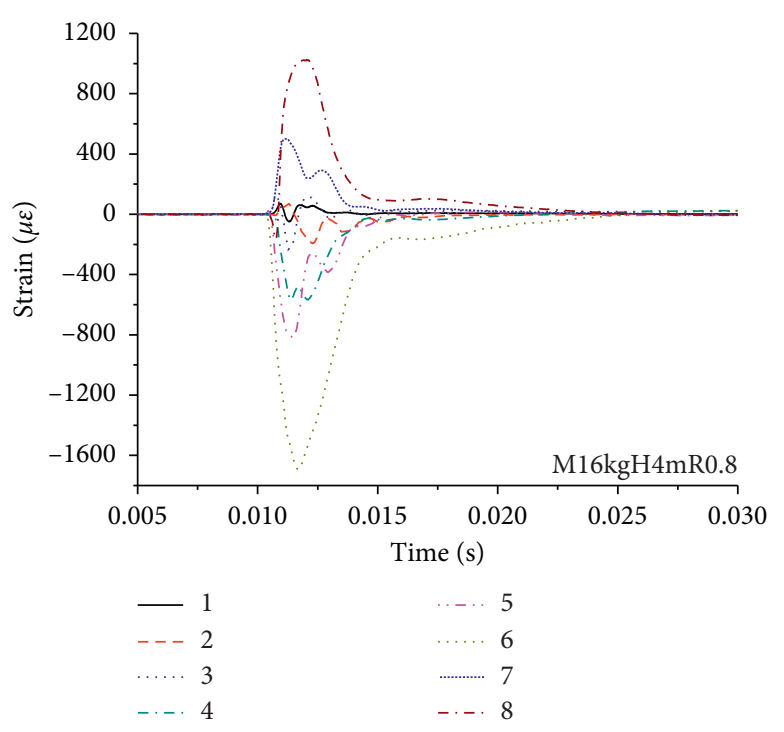

(h)

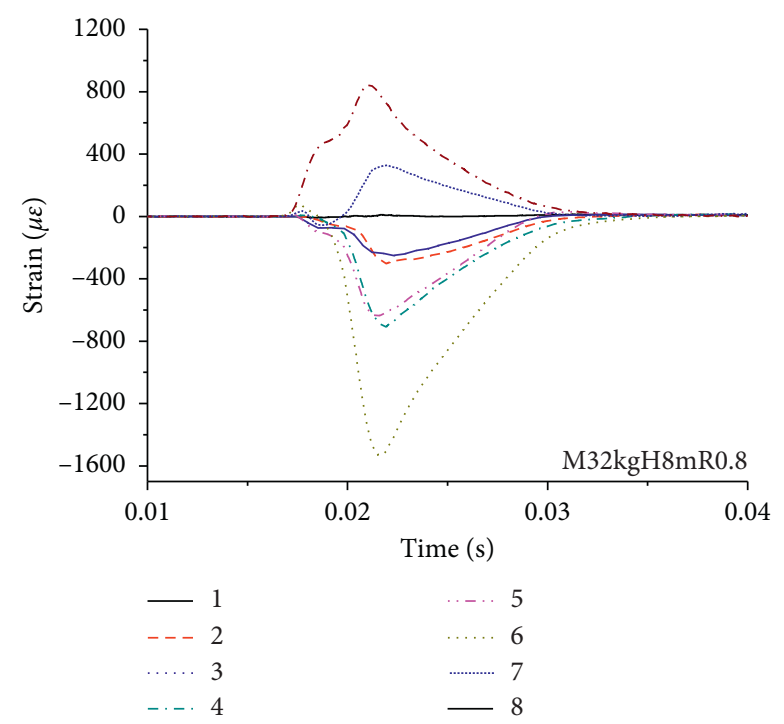

(i)

Figure 4: Strain curves of each measured point. (a) Condition 1. (b) Condition 2. (c) Condition 3. (d) Condition 4. (e) Condition 5. (f) Condition 6. (h) Condition 7. (i) Condition 8. (j) Condition 9.

has a greater circumferential strain when subjected to collapse impact load. Besides considering that there are air pressures in the natural gas pipelines, the pipelines are prone to produce axial cracks when they are subjected to impact.

By comparing the strain curves in the three columns and further analyzing the influence of soil medium on the dynamic strain of the buried pipeline under impact, it is found that the soil medium has great influence on the dynamic response of the buried pipeline under the collapse impact load. Overall, as the soil becomes denser, the slower the decay of impact energy, the greater the impact load on the pipeline and the corresponding strain. However, the looser the soil, the collapse impact energy will be absorbed by the soft soil more easily, and the influence of impact load on the pipeline will be smaller. By comparing the three transverse strain curves and analyzing the influence of drop weight on the strain curve, it is found that the weight of the drop hammer has a significant effect on the recovery cycle of strain. The recovery time of strain caused by the impact of $32 \mathrm{~kg}$ drop hammer is close to $20 \mathrm{~ms}$, while the recovery time of strain of $8 \mathrm{~kg}$ and $16 \mathrm{~kg}$ drop hammer is basically within $15 \mathrm{~ms}$.

2.2.2. Range Analysis of the Influence Factors of Pipeline Strain. In the previous section, the strain law of the pipeline subjected to impact loading was analyzed, and the differences in strain curves caused by different soils and drop weights were elaborated. This section will quantitatively analyze the influence of the soil, drop weight, and collapse height on the dynamic strain of the pipeline and 
derive the weight of three factors on the pipeline strain, which will provide reference for future engineering practice.

First, the circumferential strain at the center point of the pipe, which is the maximum value of all the measuring points, is used as the evaluation index, and the maximum strain measured in the pipe under each test condition is filled in the table. Then we get the values of $K_{1}, K_{2}$, and $K_{3}$ by summing up the strain values at every level of the influence factors, and the average values of $\bar{K}_{1}, \bar{K}_{2}$, and $\bar{K}_{3}$ are obtained further (as shown in Table 4). Finally, according to the results in the table, the trend diagram of the three factors on the pipeline strain (Figure 5) is drawn, and the main and secondary factors affecting the strain of the pipe can be discharged according to the extreme difference.

In the figure, the three polylines $M, H$, and $R$ correspond to the influence of falling weight, collapse height, and soil conditions on pipe strain. It can be clearly seen from the trend figure of strain that falling weight and collapse height have a significant effect on pipe strain. That is, as the mass of falling weight increases or the collapse height increases, the pipe strain increases. With the change of the mass of the drop hammer, the measured strain range reaches $231.80 \mu \varepsilon$, and the corresponding strain range under the influence of the collapse height is $198.46 \mu \varepsilon$. But considering the design level of each factor in the experiment, the drop weight is $8 \mathrm{~kg}, 16 \mathrm{~kg}$, and $32 \mathrm{~kg}$ doubled in turn, and the three levels of collapse height are $4 \mathrm{~m}, 6 \mathrm{~m}$, and $8 \mathrm{~m}$. The collapse height has a greater influence on the pipe strain than the drop hammer. The further observation of Figure 4 shows that the soil property has a greater influence on the strain of the buried pipeline affected by the collapse impact; that is, with the change of the soil property, the measured strain of the pipe has a violent change, and the strain difference can reach to $945.83 \mu \varepsilon$. Therefore, according to the trend figure, it can be found that the most significant factors affecting the strain of buried pipelines under the collapse impact load are the soil quality, followed by the height of the collapse, and the final is mass of the drop weight.

\subsubsection{Variance Analysis on the Influence Factors of Pipeline} Strain. ANOVA [12] is a method to test whether the mean of each group is equal under F-assumption, so as to determine whether the effect of each factor is significant. The significance level $\alpha$ is taken as $0.01,0.05$, and 0.1 . When $F \geq F_{0.01}$, it means that the influence of this factor on the index is particularly significant. When $F_{0.05} \leq F \leq F_{0.01}$, it means that the influence of this factor on the index is significant. When $F_{0.1} \leq F \leq F_{0.05}$, it means that the factor is influential but not particularly significant. When $F \leq F_{0.1}$, it means the factor is not significant. The $F_{0.01}, F_{0.05}$, and $F_{0.1}$ values are the $F$ values at the significance levels of $0.01,0.05$, and 0.1 , respectively.

By consulting the $F$ distribution critical table, the following can be obtained:

$$
\begin{aligned}
F_{0.01}(2,2) & =99.01, \\
F_{0.05}(2,2) & =19.0, \\
F_{0.1}(2,2) & =9.0 .
\end{aligned}
$$

The results of the listed calculations are shown in Table 5. $F_{\mathrm{M}}, F_{\mathrm{H}}$, and $F_{\mathrm{R}}$ represent the $F$ values of the mass of the falling hammer, the collapse height, and the pipe-soil stiffness ratio, respectively. Then, comparing with the $F$ distribution critical, we get $F_{\mathrm{M}}=1.52<F_{0.1}(2,2)$ and $F_{\mathrm{H}}=$ $1.52<F_{0.1}(2,2)$, which shows that the influence of drop weight and collapse height on pipeline strain is not significant. However, $F_{0.05}(2,2)<F_{\mathrm{R}}=29.26<F_{0.01}(2,2)$, it can be seen that the pipe-soil stiffness ratio has a significant effect on pipe strain. Among these three factors, the most significant factor affecting the maximum strain of the pipeline is the pipe-soil stiffness ratio. In the analysis of variance, it can be found that the level of the falling weight and the collapse height are not significant, but it can be found in the range analysis and the slope figure that the above two factors have influence on the pipe strain. Based on the above analysis, we suspect that the result of variance analysis may be because the experimental error is large and the degree of freedom of error is small, so that the sensitivity of the test is reduced.

\section{Study on Numerical Simulation of Collapse Impact}

From the analysis in the previous section, it was found that the pipe-soil stiffness ratio has a significant effect on the dynamic response of the collapse impact load on the pipeline. The numerical simulation study in this section will further explore the effect of the pipe-soil stiffness ratio on the dynamic response of the pipeline under impact.

3.1. Finite Element Model. We apply ANSYS/LS-DYNA to simulate the impact of heavy objects falling on the ground and affecting the pipeline. There are three parts in the model (as shown in Figure 6), in which the pipeline and falling hammer materials are steel, and the Johnson-Cook material model is used (Table 6). The soil material [13-15]adopts SOIL_AND_FOAM_FAILURE material model (Table 7). In order to reduce the calculation, a $1 / 4$ model was established. Fixed constraints were imposed on the two symmetry planes. The soil upper surface does not impose constraints, and the remaining surfaces were set as nonreflective boundaries to simulate an infinite soil medium. The model uses hexahedral solid elements. Automatic surface-to-surface contact is set between soil and pipes which is also set between the weight and the soil.

3.2. Numerical Simulation Results. A numerical model is established based on the parameters in the above with ANSYS-LSDYNA software. In order to verify the reliability of the numerical simulation, the depth of the three types of soil in the drop hammer test and that in the numerical 
TABLE 4: Range analysis table.

\begin{tabular}{lcccc}
\hline Condition & Mass of hammer, $M(\mathrm{~kg})$ & Height of collapse, $H(\mathrm{~m})$ & Pipe-soil stiffness ratio, $R$ & Maximum strain $(\mu \varepsilon)$ \\
\hline 1 & 16 & 6 & 1.602 & -7.602 \\
2 & 8 & 8 & 1.602 & -713.1 \\
3 & 32 & 4 & 1.215 & -621.9 \\
4 & 8 & 4 & 1.215 & -496.1 \\
5 & 16 & 8 & 1.215 & -767.3 \\
6 & 32 & 6 & 0.843 & -1073.3 \\
7 & 32 & 8 & 0.843 & -1743.8 \\
8 & 8 & 6 & 0.843 & -1534.4 \\
9 & 16 & 4 & -2336.70 & -1690.8 \\
$K_{1}$ & -2743.60 & -2808.80 & -2131.50 & -4969.00 \\
$K_{2}$ & -3254.60 & -3224.20 & -778.90 & \\
$K_{3}$ & -3439.00 & -3404.20 & -710.50 & \\
$\bar{K}_{1}$ & -914.53 & -936.27 & -1656.33 \\
$\bar{K}_{2}$ & -1084.87 & -1074.73 & & \\
$\bar{K}_{3}$ & -1146.33 & -1134.73 & & \\
\hline
\end{tabular}

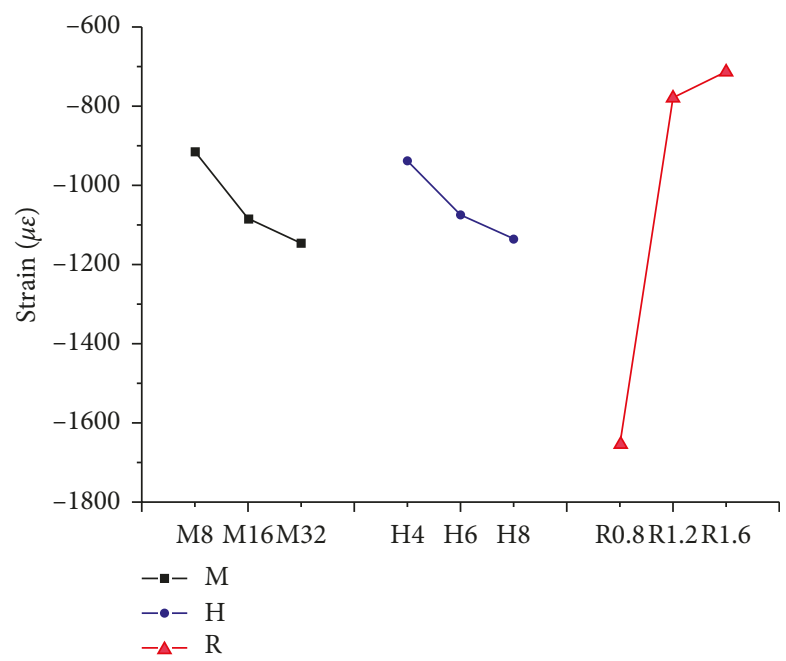

Figure 5: Slope diagram of strain influence factors.

TABLE 5: Variance analysis table.

\begin{tabular}{|c|c|c|c|c|c|}
\hline Source of variance & Square sum of factor deviations & Degree of freedom & Mean square deviation & $F$ & Significant level \\
\hline Mass of hammer, $M$ & 86522.84 & 2 & 43261.42 & 1.52 & Not significant \\
\hline Height of collapse, $H$ & 62162.04 & 2 & 31081.02 & 1.09 & Not significant \\
\hline Pipe-soil stiffness ratio, $R$ & 1669169.00 & 2 & 834584.3 & 29.26 & Significant \\
\hline Error & 57055.72 & 2 & 28527.86 & & \\
\hline
\end{tabular}

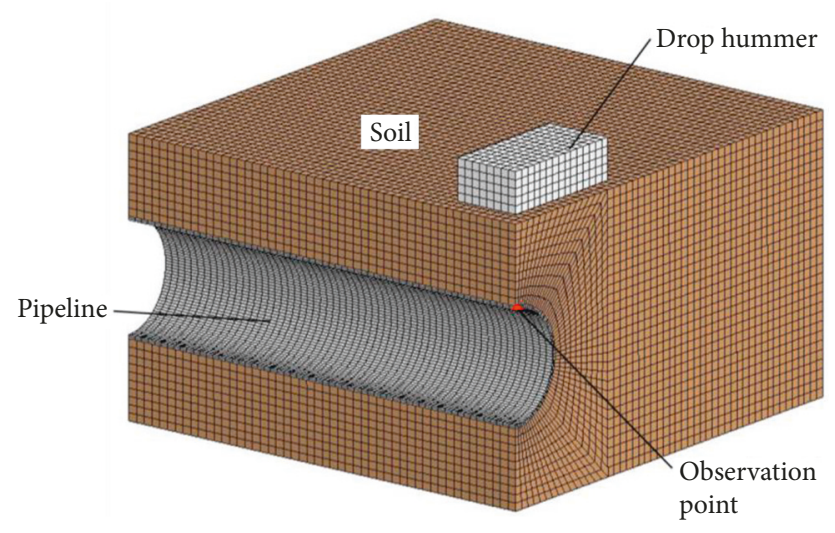

FIgURE 6: Numerical calculation model. 
TABLE 6: Q235 material parameters.

\begin{tabular}{lccccccc}
\hline \multirow{2}{*}{ Material model } & Density $\left(\mathrm{g} / \mathrm{cm}^{3}\right)$ & Shear modulus G0 & Cutoff pressure PC & \multicolumn{4}{c}{ Material constant } \\
& & & $A(\mathrm{MPa})$ & $B(\mathrm{MPa})$ & $n$ \\
\hline Johnson-Cook & 7.85 & 0.77 & -9 & 650.0 & 439.0 & 0.503 & 0.1 \\
\hline
\end{tabular}

TABLE 7: Material parameters of soil [16-18].

\begin{tabular}{lccccccccc}
\hline Parameters & R0 & G & Bulk & A0 & A1 & A2 & PC & VCR & Unit \\
\hline Value & 1.84 & $6.385 E-4$ & $3.00 E-1$ & $3.4 E-13$ & $7.033 E-7$ & 0.30 & $-6.90 E-8$ & 0.0 & g-cm- $\mu$ s \\
Parameters & EPS2 & EPS3 & EPS4 & EPS5 & EPS6 & EPS7 & EPS8 & EPS9 & EP10S \\
Value & -0.104 & -0.161 & -0.192 & -0.224 & -0.246 & -0.271 & -0.283 & -0.290 & -0.4 \\
Parameters & P2 & P3 & P4 & P5 & P6 & P7 & P8 & P9 & P10 \\
Value & $2.0 E-4$ & $4.0 E-4$ & $6.0 E-4$ & $1.2 E-3$ & $2.0 E-3$ & $4.0 E-3$ & $6.0 E-3$ & $8.0 E-3$ & $4.1 E-2$ \\
\hline
\end{tabular}

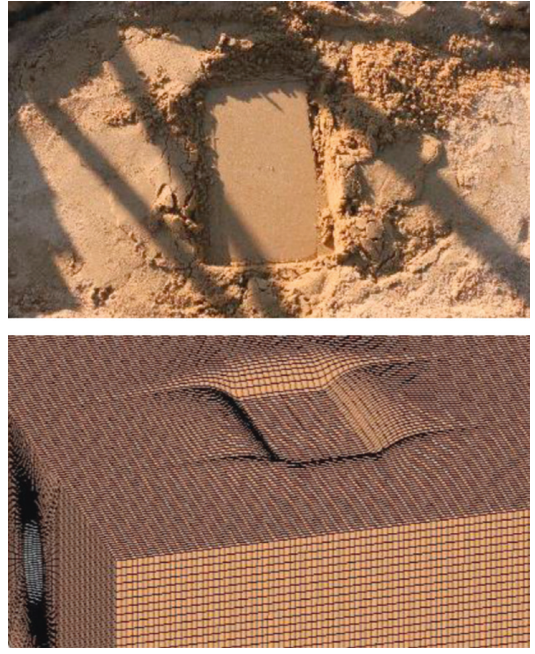

(a)
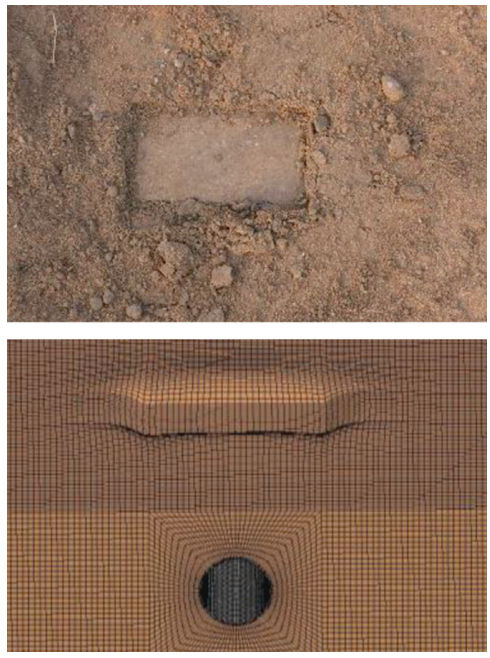

(b)
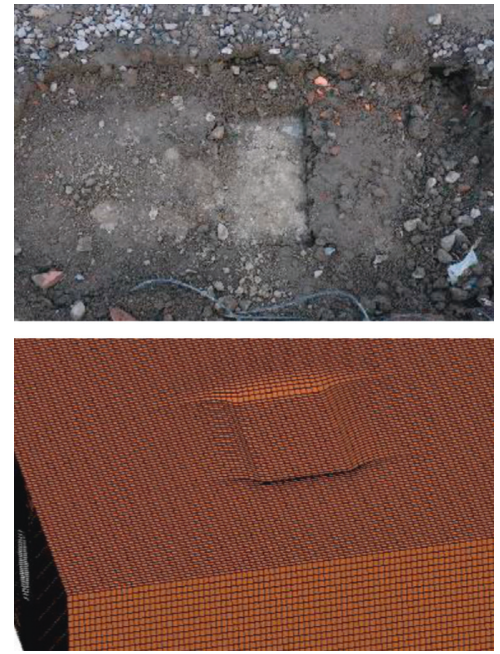

(c)

Figure 7: Comparison of deformation between test and numerical simulation. (a) Comparison of working condition 2. (b) Comparison of working condition 5. (c) Comparison of working condition 8.

simulation are compared. We selected the working conditions 2, 5, and 8 of the experimental scheme in the paper for numerical simulation (as marked in Table 3). The deformation of the test and numerical simulation is shown in Figure 7. The depth of these pits and errors are shown in Table 8 .

Through the comparative analysis of soil pit depth, it is found that the maximum error between the numerical simulation results and the test results is within $20 \%$, and the average error is $16.4 \%$. It can be considered that the numerical simulation is correct and reliable.

The stress cloud diagram in the impact process is obtained through calculation, as shown in Figures 8 and 9. The stress cloud diagram in Figure 8 shows the stress propagation process at the moment of collapse weight impacting the ground. From the figure, the law of stress propagation can be seen intuitively. The stress is stable at the initial propagation and remains around $2.55 \mathrm{MPa}$. When the stress wave is transferred to the buried pipeline, the peak value of the stress wave increases obviously, and the peak value of the stress wave on the pipe is more than $15 \mathrm{MPa}$. The stress wave
Table 8: The depth of pits and errors.

\begin{tabular}{lcccc}
\hline Condition & 2 & 5 & 8 & Average \\
\hline Depth of pit in test $(\mathrm{cm})$ & 2.10 & 1.80 & 1.20 & - \\
Depth of pit in simulation $(\mathrm{cm})$ & 2.45 & 2.04 & 1.43 & - \\
Error (\%) & 16.7 & 13.3 & 19.2 & 16.4 \\
\hline
\end{tabular}

on the pipe in Figure 9 also shows that the stress wave is obviously enhanced when the stress wave is transmitted to the pipe in the soil. Therefore, the influence of the collapse vibration on the buried pipeline in the demolition project must be highly valued.

3.3. Analysis of Numerical Simulation Results. According to the calculation formula of pipe-soil stiffness ratio, by changing the deformation modulus of the soil medium in the numerical calculation model, the stress and strain results of the buried pipe under the conditions of five different pipesoil stiffness ratios are obtained. The corresponding situation of deformation modulus and pipe-soil is shown in Table 9. 

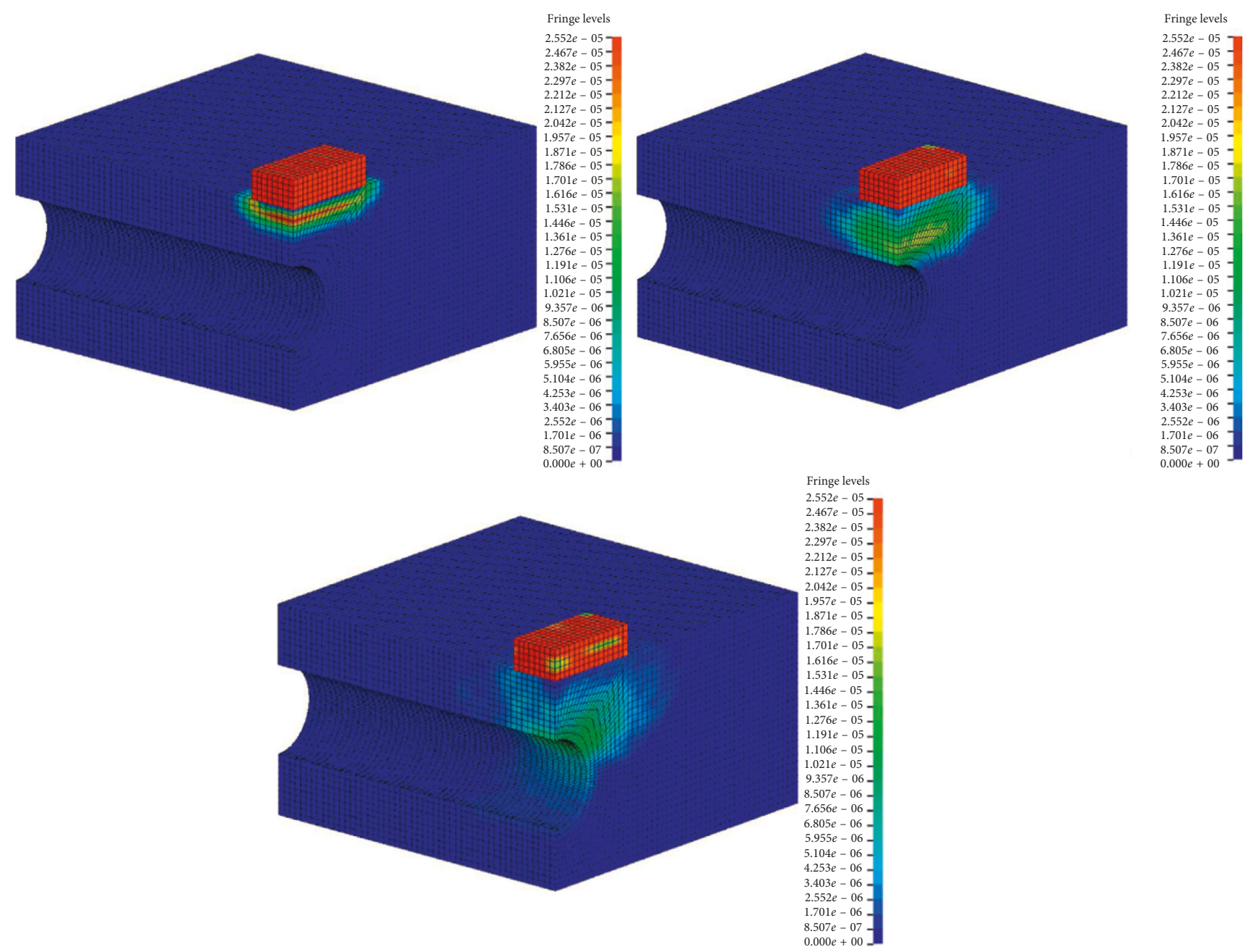

Figure 8: Stress cloud map of the impact process.
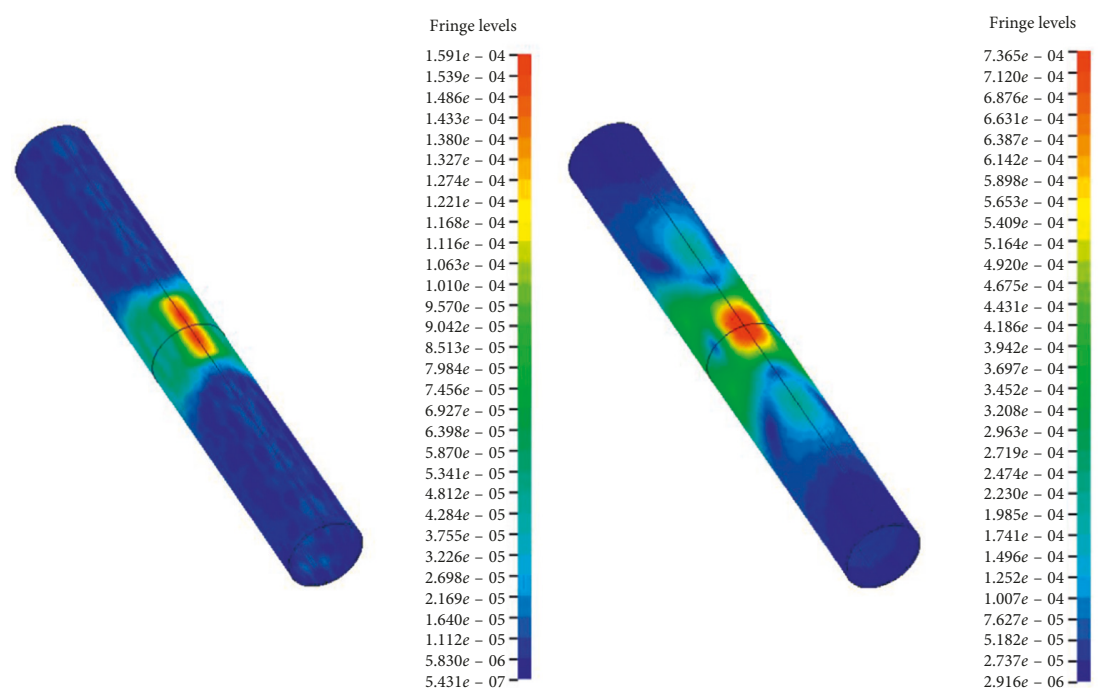

Fringe levels

$7.424 e-04$

Figure 9: Stress cloud map of pipe.

From the table, it can be seen that with the decrease of the deformation modulus of the soil medium, the pipe-soil stiffness ratio is greater.
The center point of the pipe in the numerical model is selected as the observation point (Figure 6). We analyze the effective plastic strain and the effective plastic stress at this 
TABle 9: Corresponding relation between soil deformation modulus and pipe-soil stiffness ratio.

\begin{tabular}{lccccc}
\hline $\begin{array}{l}\text { Deformation modulus of } \\
\text { soil (MPa) }\end{array}$ & 59.26 & 47.38 & 39.51 & 33.86 & 29.63 \\
\hline Pipe-soil stiffness ratio & 1.00 & 1.25 & 1.5 & 1.75 & 2.00 \\
\hline
\end{tabular}

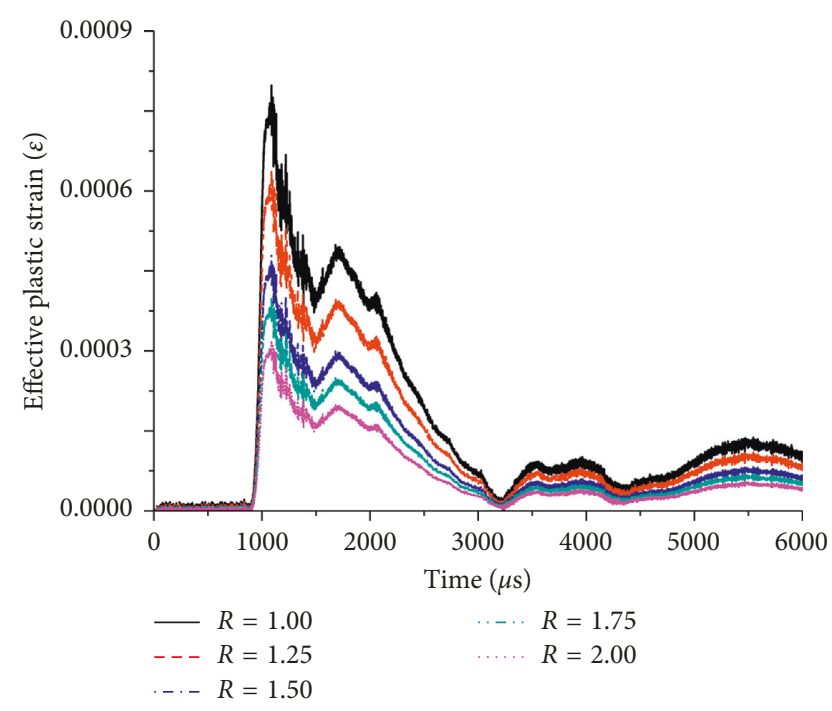

Figure 10: Comparison of effective plastic strain.

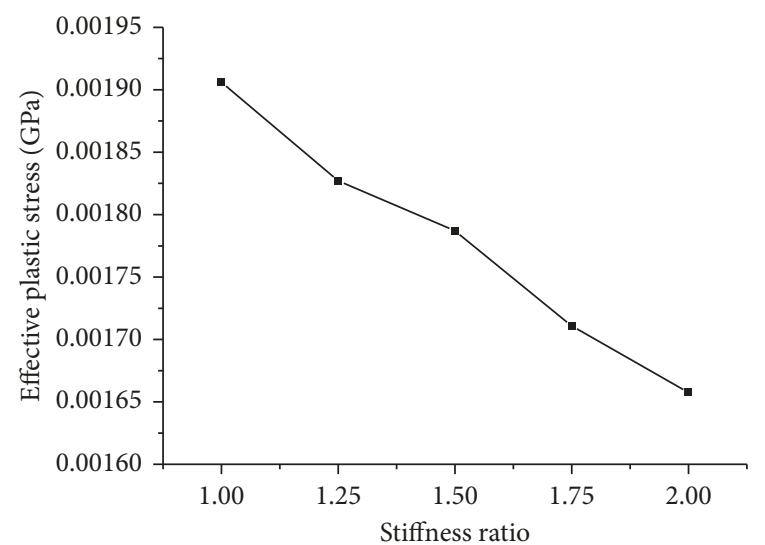

Figure 11: Variation trend of effective plastic stress with pipe-soil stiffness ratio.

point. The images of the effective plastic strain of the observation point under five types of stiffness ratios over time are plotted (Figure 10). The trend image of peak effective plastic stress with tube stiffness ratio is also plotted (Figure 11). From Figure 10, it can be found that the effective plastic strain-time curves under five kinds of pipe-soil stiffness ratio are basically similar. The strain curves of the numerical simulation are similar to those of the measured strain wave, which proves the validity of the numerical simulation again. By comparing the final plastic strain platform of 5 kinds of pipe-soil stiffness ratio, it is found that the plastic strain of pipe is smaller with the increase of the pipe-soil stiffness ratio. From Figure 11 of peak effective plastic stress along with the pipe-soil stiffness ratio, the above conclusion is also proved. With the increase of pipesoil stiffness ratio, the peak effective plastic stress at the observation point also showed a downward trend. The main reason for this result is that as the soil becomes looser, after the weight hits the ground, the kinetic energy is more converted into the internal energy of the soil deformation, and the energy ultimately transmitted to the pipeline is reduced. Finally, the stress and strain of the pipeline have a significant reduction.

\section{Conclusion}

Through the above study, the main conclusions are drawn as follows:

(1) After the buried pipeline is subjected to collapse impact load, the hoop strain at each measurement point is greater than the axial strain, and cracks along the axial direction are easily generated in the case of a prepressure inside the pipeline.

(2) Through the range analysis and variance analysis, the pipe-soil stiffness ratio is the most significant factor influencing the dynamic strain of buried pipelines among the mass of weight, the height of collapse, and the pipe-soil stiffness ratio.

(3) Numerical simulation shows that under the condition of unchanged buried pipeline properties, the more looser the soil is, the smaller the plastic stress and strain of the buried pipeline, and influence of the buried pipeline under the collapse impact load is smaller.

\section{Data Availability}

There are no linked research data sets for this paper. All the data supporting the conclusions of the study will be made available on readers' request. The data can be available by contacting with Mr. Wang whose e-mail address is 1257950377@qq.com

\section{Conflicts of Interest}

The authors declare that there are no conflicts of interest regarding the publication of this paper.

\section{Acknowledgments}

The research work was supported by the National Natural Science Foundation of China (Grant nos. 51508569 and 51304218). It was also supported by the Jiangsu Provincial Natural Science Foundation (Grant no. BK20151449).

\section{References}

[1] X. U. Tao-Long, X. G. Zeng, A. L. Yao, and H.-Y. Chen, "reliability analysis of buried gas pipeline under ramming impact loading," Journal of Sichuan University, vol. 44, no. 2, pp. 96-16, 2012. 
[2] C. J. Han, H. Zhang, and J. Zhang, "Effects of surface load on stress-strain characteristics of the pipeline buried in hard rock region," Journal of Safety Science and Technology, vol. 11, no. 7, pp. 65-71, 2015.

[3] B. Yan, P. Y. Lin, and H. T. Yu, "Analysis of settlement and tamping energy dissipation," Geotechnical Engineering, vol. 33, no. 1, pp. 242-243, 2011.

[4] T. Shui and Z. Wang, "Effect of impact mode on treatment effect of dynamic compaction," Rock and Soil Mechanics, vol. 29, no. 11, pp. 3119-3123, 2013.

[5] J. Zhou, S. F. Zhang, and M. C. Jia, "Theoretic research situation and latest technical progress of dynamic consolidation method," Tunnelling and Underground Space Technology, vol. 2, no. 3, pp. 510-516, 2006.

[6] Z.-M. Wang, Study on Mechanical Properties of Buried Pipelines under Traffic Load, Zhejiang University, HangZhou, China, 2006.

[7] J.-T. Yang, Longitudinal Mechanical Properties of Buried Pipelines under Vertical Loads, Zhejiang University, HangZhou, China, 2006.

[8] Z.-J. Sun, Analysis of Mechanical Properties of Buried Pipelines under Ground Surcharge Load, Zhejiang University, HangZhou, China, 2014.

[9] M. Liu and M. Yang, "Modeling the behavior of natural gas pipeline impacted by falling objects," Engineering Failure Analysis, vol. 42, pp. 45-59, 2014.

[10] A. M. Gresnigt and S. A. Karamanos, "Response of steel tubes under concentrated lateral loads," Steel Construction, vol. 7, no. 2, pp. 133-140, 2014.

[11] A. Mosadegh and H. Nikraz, "Buried pipe response subjected to traffic load experimental and numerical investigations," International Journal of Geomate, vol. 13, no. 39, pp. 1-8, 2017.

[12] L. Ying-Hua and M.-Y. Zhang, "Sensitivity analysis of the influence factors on slope excavation," Journal of Civil Engineering and Management, vol. 34, no. 1, pp. 113-116, 2017.

[13] C. Xu and W. Feng-Hui, "Study on the process of different head shape projectiles penetrating into soil," Science Technology and Engineering, vol. 11, no. 15, pp. 3435-3438, 2011.

[14] B. Zhou, "Numerical simulation of soil cutting by biaxial vertical spiral trenching device," Hubei Agricultural Sciences, vol. 52, no. 8, pp. 1938-1942, 2013.

[15] Z. F. Zhang, F. Hao, and Z. X. Feng, "FEM analysis of vibration drum-soil model," Construction Machinery, vol. 21, no. 15, pp. 89-93, 2008.

[16] J. Wang, "Simulation of landmine explosion using LS-Dyna3d software: benchmark work of simulation of explosion in soil and air," Defence Science \& Technology Organisation, vol. 14, no. 1, pp. 15-19, 2001.

[17] J.-H. Zhao, "Numerical analysis of explosion in soil," Journal of Architectural Science and Engineering, vol. 28, no. 1, pp. 96-99, 2011.

[18] J.-Y. Fan, "Numerical analysis of load on shallow buried structure in soil under explosive condition," in Proceedings of National Symposium on Safety and Protection of Engineering Structures, Beijing, China, September 2005. 


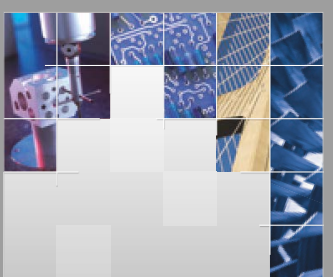

\section{Enfincering}
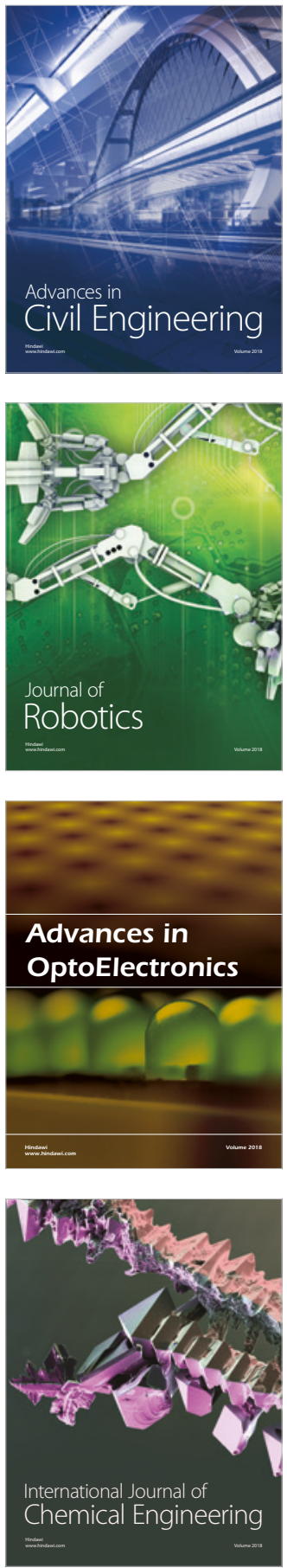

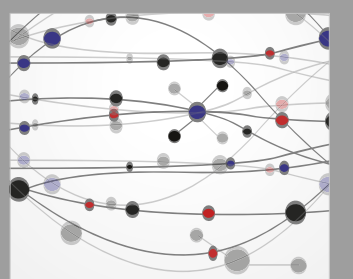

\section{Rotating \\ Machinery}

The Scientific World Journal

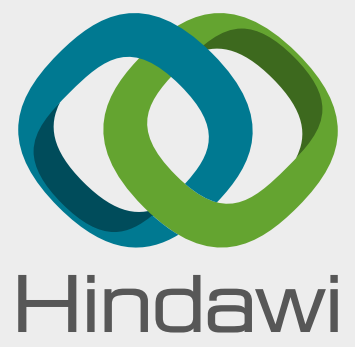

Submit your manuscripts at

www.hindawi.com
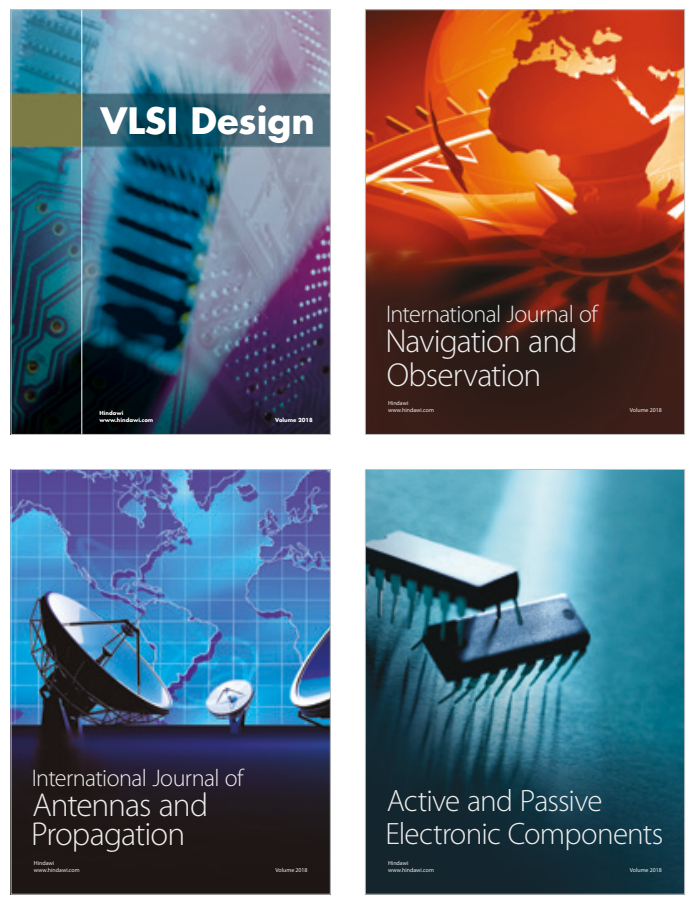
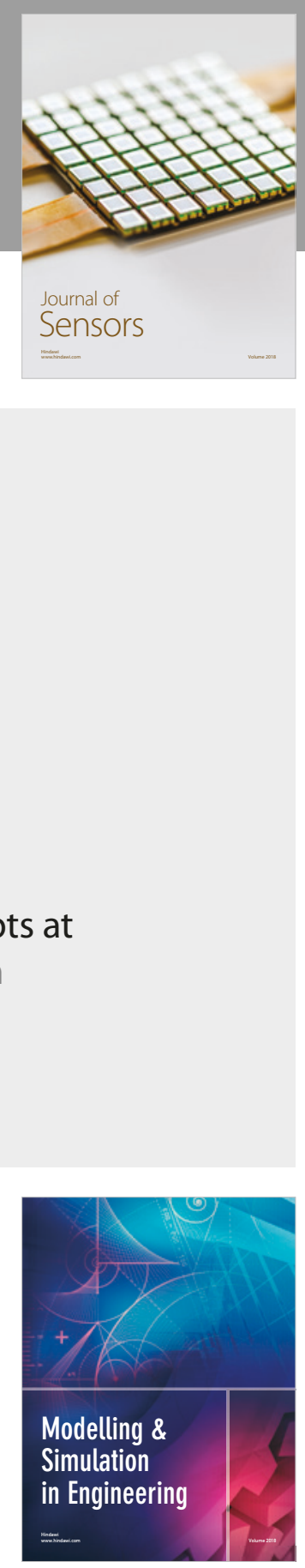

\section{Advances \\ Multimedia}
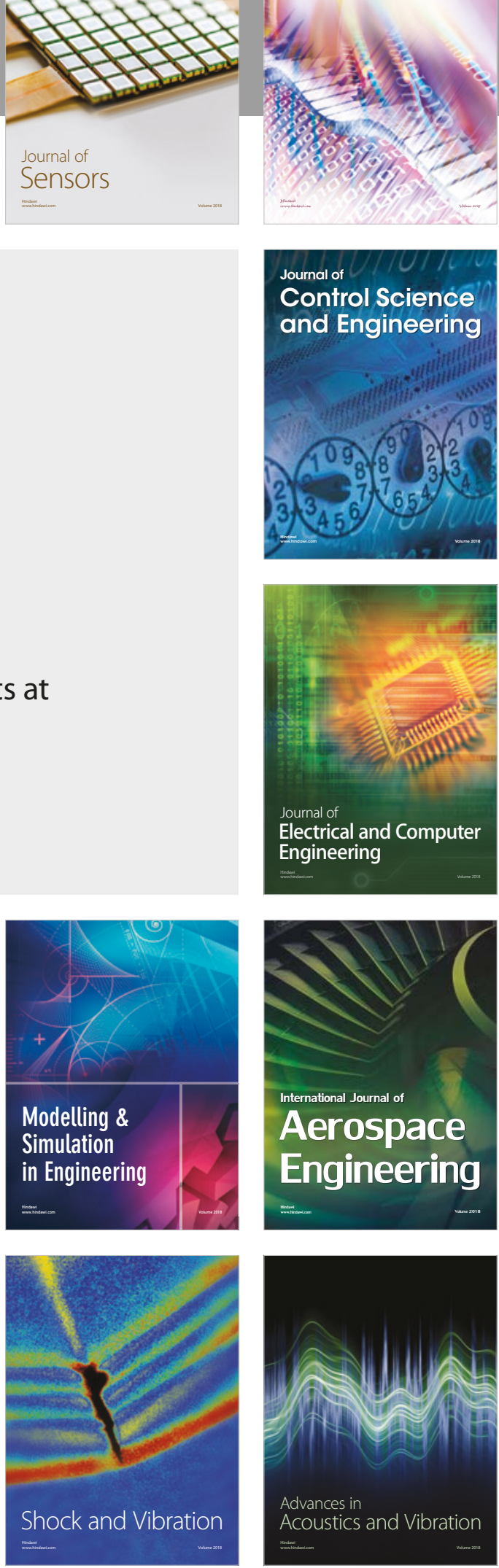